\title{
In-Flight Dark Current Nonuniformity Used for Space Environment Model Benchmarking
}

\author{
C. Inguimbert ${ }^{(}$, S. Bourdarie, M. Beaumel, M. C. Ursule ${ }^{(\mathbb{D}}$, and R. Ecoffet
}

\begin{abstract}
In-flight dark current nonuniformity measurements performed on three different satellites [JASON-2, SPRINT-A, and SAT-X (name withheld)] are compared to ground calculations. The standard deviation of the DCNU is calculated using both the AP8 and AP9 environment models. These predictions are compared to some calculations made with proton fluxes measured with the Influence sur les Composants Avancés des Radiations de l'Espace-NG spectrometer embedded on the JASON-2 satellite. The reliability of AP8 and AP9 models is analyzed. AP9 model appears to consistently overestimate the in-flight degradation.
\end{abstract}

Index Terms-Charge-coupled device (CCD) active pixel sensor (APS), dark current nonuniformity (DCNU), displacement damages, image sensors, space environment.

\section{INTRODUCTION}

$\mathbf{S}$ OLID-STATE sensor arrays such as charge-coupled devices (CCDs) or CMOS image sensors (CIS), see their properties degrading continuously during the missions due to the radiative space environment [1]. Of particular interest is the cumulative increase in dark current induced by atomic displacements [1]. Both protons and electrons in the radiation belts can produce some significant degradation by displacement damage [2]. The distribution of the dark current from pixel to pixel over a pixel array, referred to as the dark current nonuniformity (DCNU) is particularly sensitive to such kind of phenomenon. The spread of this function can be simply estimated with the standard deviation (SD) of the distribution. This parameter which is the main focus of this paper will be called SD of the DCNU ( $\left.\mathrm{SD}_{\mathrm{DCNU}}\right)$.

The trapped protons of the radiation belts represent the main constraint at low earth orbits while trapped electrons can dominate displacement damage effects at geostationary orbit. Energetic protons of some tens of million electronvolts introduce defects in the semiconductors by means of Coulombic and nuclear interactions (elastic, inelastic) [3]. The increase in DCNU is particularly troublesome for systems such as star trackers. The presence of highly degraded pixels ("hot pixels") affects the performances of such kind of low-noise systems.

C. Inguimbert, S. Bourdarie, M. C. Ursule are with the DESP, ONERA, 31055 Toulouse, France (e-mail: christophe.inguimbert@onera.fr).

M. Beaumel is with SODERN, 94451 Limeil-Brevannes cedex, France (e-mail: matthieu.beaumel@sodern.fr).

R. Ecoffet is with the CNES (French space agency), 31400 Toulouse, France (e-mail: Robert.Ecoffet@cnes.fr).
SODERN [4], a company specialized in designing and manufacturing of space instrumentation, monitors in certain cases, the SD of the in-flight DCNU. These onboard feedback data is an interesting source of information for the radiative space environment survey. The comparison of the prediction with inflight measurements can serve to the validation of proton environment specification models. This is what is proposed here.

This paper is part of a continuing effort by ONERA and CNES to improve space environment models. It follows up previous publications [6], [7], where direct distance dialing, total ionizing dose (TID), and single event upsets (SEUs) data have been used to investigate uncertainties in trapped proton specification models. The SEU rate is recorded by an error detection and correction (EDAC) counter, but also the current of a light emitting diode of an optically stimulated luminescence dosimeter (OSL) and the TID degradation of a RadFET has been investigated in [6] and [7]. This paper is based on the use of the environment data provided by the "Influence sur les Composants Avancés des Radiations de l'Espace" (ICARE) radiation monitor, which was embarked in 2000 onboard the Argentinean satellite SAC-C [8]. An upgraded version (ICARE-NG) of this equipment is also embedded on the JASON-2 satellite [9], allowing some further studies.

In this paper, the $\mathrm{SD}_{\mathrm{DCNU}}$ of a star tracker's $\mathrm{CCD}$ is chosen as the parameter of interest. The SED16/26 star tracker from SODERN is embedded on three different satellites (JASON-2, SPRINT-A, and SAT-X) for which the SD of the in-flight DCNU is monitored and available for different periods of several months. It should be noted that for confidentiality reasons the name of the third project will not be given here. The SED16/26 star tracker is based on the CCD 47-20 from e2v [5], a three-phase, and buried channel CCD that is the focus of this paper. The proton fluxes encountered by the CCD 47-20 is evaluated using the ICARE-NG data and both AP8, and AP9 models. The $\mathrm{SD}_{\mathrm{DCNU}}$ of this CCD is then evaluated for different environment data (AP8, AP9, and ICARE-NG) and for the three different missions. The comparison to in-flight data is made to assess the reliability of the AP8 and AP9 proton environment models.

Section I presents how the proton fluxes have been estimated for JASON-2 and SPRINT-A satellites using the JASON-2/ICARE-NG radiation monitor. The comparison with AP8 and AP9 models will be shown. Then in Section II, will be presented, the calculation of the $\mathrm{SD}_{\mathrm{DCNU}}$. To finish the calculated $\mathrm{SD}_{\mathrm{DCNU}}$ will be compared to in-flight data for the three different missions. 


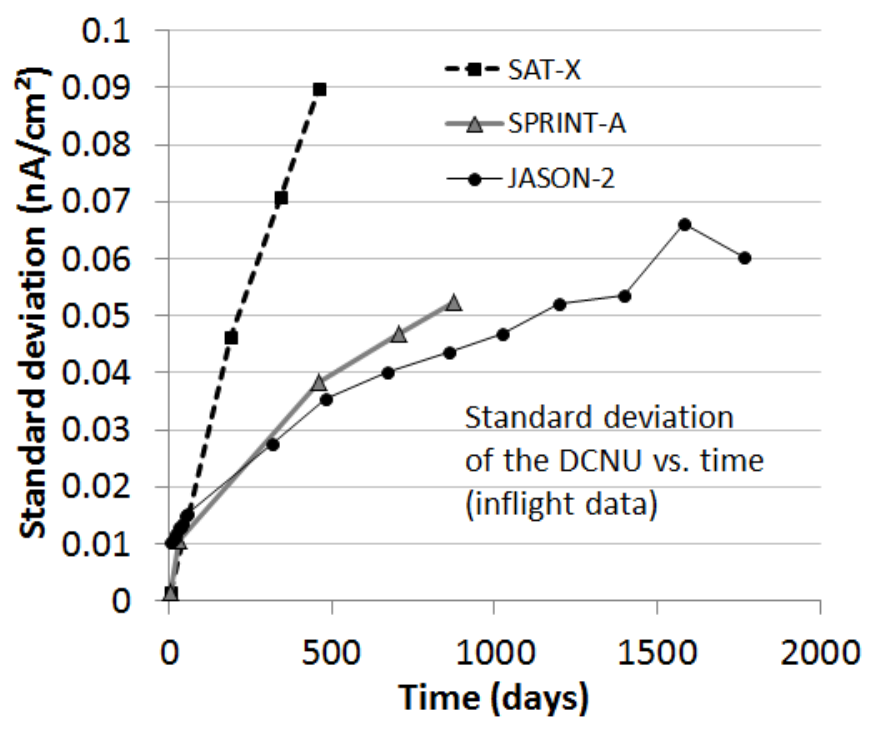

Fig. 1. SDDCNU versus time for the three different missions JASON-2, SPRINT6A, and SAT-X.

\section{SELECTED Missions}

This paper has been carried out with three different missions. On each of these missions, an SED16/26 star tracker manufactured by SODERN is embedded and the $\mathrm{SD}_{\mathrm{DCNU}}$ is measured over time. This equipment presents several features which makes it well suited for dark current measurements: its CCD includes an area shielded from light on which DCNU can be characterized, and the CCD is maintained at a constant temperature of $-10{ }^{\circ} \mathrm{C}$, thanks to thermo-electric cooling. The DCNU is automatically estimated during start tracker self-test by measuring twice the pixel values of a column of 1000 pixels located under the shielded area of the CCD, using integration times of, respectively, $1 \mathrm{~ms}$ and $5 \mathrm{~s}$. Dark current is obtained by performing a pixel-wise subtraction between the values measured at $5 \mathrm{~s}$ and $1 \mathrm{~ms}$, which allows for suppression of the electrical offsets. The SD of the resulting values is then computed.

The first mission is the low earth orbit JASON-2 satellite (1336 $\mathrm{km}$ altitude and $66^{\circ}$ inclination). The second mission is a communications satellite, SAT_X, launched in 2012. The satellite is on a transfer orbit reaching an apogee of $5000 \mathrm{~km}$ in August 2012 and then decreases over time to $3500 \mathrm{~km}$ in March 2015. The perigee of this orbit is of $265 \mathrm{~km}$. The inclination of the orbit is maintained between $49.9^{\circ}$ and $45.95^{\circ}$. The third mission is a low earth orbit Japanese mission, SPRINT-A, launched on September 14, 2013. Its apogee is $1160 \mathrm{~km}$, its perigee is maintained at $960 \mathrm{~km}$ and its inclination is very low $\left(29.7^{\circ}\right)$. The $\mathrm{SD}_{\mathrm{DCNU}}$ measured on these three missions is presented in Fig. 1. Even if these orbits are in a proton rich environment, the different mission profiles lead to relatively different increase in the $\mathrm{SD}_{\mathrm{DCNU}}$. The most constraining orbit is clearly the one of the SAT-X satellites which presents fluxes two times larger than the two other missions. Note that the peak at 1582 days of the DCNU of JASON-2 seems to be erroneous. External factors such as blooming from parasitic light or transient signal from protons can sometimes affect the measurements.

\section{Calculation of the Proton FluXes}

\section{A. Rationale for Focusing on Proton Flux}

On CCDs exposed to radiation damage, one of the main degradation mechanisms is the increase of dark current and DCNU originating from surface traps created at the $\mathrm{Si}-\mathrm{SiO}_{2}$ interface by ionization, and from bulk atomic defects created by displacement damage in the pixel depletion zone [1]. The SED16/26 star tracker uses several techniques to reduce the dark current generation of surface states. First, the interface between the n-doped channel and the oxide surface is driven in inversion during the integration time, filling the surface traps with holes and preventing the generation of electron-hole pairs. Second, dither clocking is used [10], which consists in quickly transferring charge packets back and forth between the phases during integration. As the CCD 47-20 does not feature a multipinned phase implant [11], one of the phases cannot be inverted during the integration time. However, as charges are transferred at a faster rate than the holes emission time constant of the interface traps, the surface effectively remains inverted. In consequence, surface dark current is greatly reduced and TID has a very limited effect on the DCNU increase of the CCD 47-20. This has been confirmed by Cobalt-60 TID tests performed on CCD 47-20 flight lots: after an exposure of $7.5 \mathrm{krad}$, the DCNU was observed to increase only by $25 \%$ when dither clocking was used. In this paper, the most constraining mission with regards to TID is SAT-X, with an exposure of $8.4 \mathrm{krad}$ at CCD level. In Section II, the DCNU was shown to increase by a factor higher than 60 on this mission. Electron and proton-induced ionization are considered to contribute to less than $1 \%$ of the measured DCNU.

Therefore, the DCNU increase observed in-flight can be considered as being directly linked to atomic displacement defects created in the bulk of the star tracker CCD. According to the altitudes of spacecraft of concern, the displacement damage is assumed to be induced only by energetic protons. Electron contribution can, at first order, be neglected at such low altitudes. Such a hypothesis is justified by the absence of relativistic electrons in the inner zone as reported by Fennel et al. [12]. The trapped proton environment is deduced from environment specification models, i.e., AP8min and AP9 (Mean V1.30.001) and from in situ measurements from the ICARE-NG radiation monitor embedded on JASON-2 satellite. JASON-2/ICARE-NG proton fluxes will serve as comparison reference to assess the reliability of AP8 and AP9 model. This comparison will be performed of course for JASON-2 satellite but also for SPRINT-A. Because SPRINT-A is at lower altitude than JASON-2, it makes it possible to perform a projection of the JASON-2/ICARE-NG measurements on the orbit of the former. But this task is not possible with the third satellite (SAT-X) that evolves on a transfer orbit that crosses different orbits at different altitudes. The projection procedure is described in Section III-B. In this section, the average fluxes received by the CCD 47-20 on different orbits are presented (Section III-C). The transport of the incident fluxes through the shielding down to the CCD is described in Section III-D. 


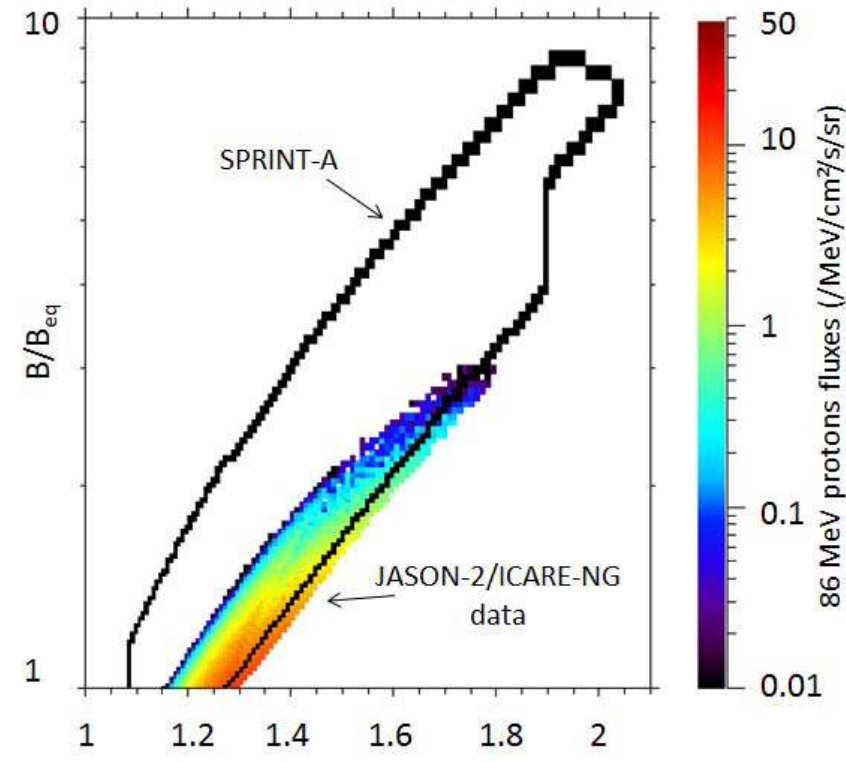

Fig. 2. B/Beq versus L map. Color coded is JASON-2/ICARE_NG 86-MeV proton differential flux averaged during January 2014. White area above color area is the loss cone, while the white area below the color area corresponds to locations above JASON-2 altitude. The black curve delineate the area where SPRINT-A is located during January 2014.

\section{B. Projection of JASON-2/ICARE-NG Data to the SPRINT-A Orbit}

Trapped particles in the radiation belts are well known to be organized according to $\mathrm{L}$ and $\mathrm{B} / \mathrm{B}_{\mathrm{eq}}$, where $\mathrm{L}$ denotes a field line, $\mathrm{B}$ is the magnetic field strength at a given point along the field line $\mathrm{L}$, and $\mathrm{B}_{\mathrm{eq}}$ is the magnetic field strength at the magnetic equator along the same field line. Given a magnetic field model, it is then possible to compute for any location (altitude, latitude, and longitude) the corresponding $\mathrm{L}$ and $\mathrm{B} / \mathrm{B}_{\mathrm{eq}}$. In this paper, IGRF-12 plus Olson-Pfitzer quiet magnetic field model is selected. Fig. 2 provides JASON-2/ ICARE_NG $86 \mathrm{MeV}$ proton differential flux averaged during January 2014 in a B/B eq versus L map. White area above color area is the loss cone, while the white area below the color area corresponds to locations above JASON-2 altitude. The black curve delineates the area where SPRINT-A is located during January 2014. Where SPRINT-A does see trapped protons (colored area inside black curve) the protons fluxes can be deduced from JASON-2/ICARE-NG measurements.

To deduce SPRINT-A trapped proton fluxes from $20 / 09 / 2013$ to $21 / 08 / 2015$, a B/B eq versus $\mathrm{L}$ map is computed for all proton energy channels available onboard JASON-2/ ICARE_NG every month. Then SPRINT-A is flown in this map where altitude, latitude, and longitude are first converted into $\mathrm{B} / \mathrm{B}_{\mathrm{eq}}$ and $\mathrm{L}$. The average spectrogram thus computed is shown on Fig. 3(b), the other satellites are shown in Fig. 3(a) and (c).

\section{Incident Proton Fluxes, AP8, AP9, and ICARE-NG}

The altitude, latitude, and longitude of JASON-2, SPRINT-A, and SAT-X satellites versus time have been computed running SGP4 NORAD's two lines elements propagator with a time step of $20 \mathrm{~s}$. It ensures us to fly the real orbit in
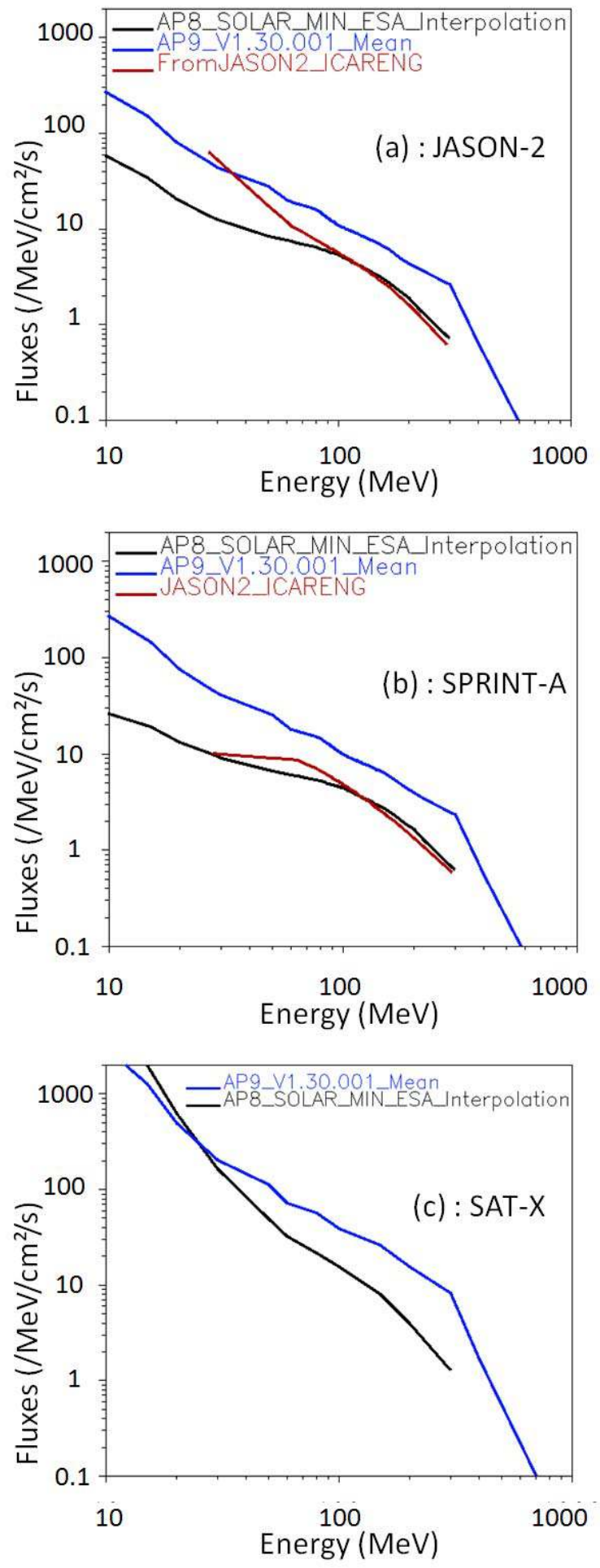

Fig. 3. Proton fluxes encountered by the three satellites (a) JASON-2, (b) SPRINT-A, and (c) SAT-X during their missions, evaluated according to the AP8 min, AP9 Mean V1.30.001, and JASON-2/ICARE_NG measurements.

the engineering specification models. Then the trapped proton environment was calculated along different orbits with a 20-s time step from the AP8 min and AP9 Mean V1.30.001 models as well as from the JASON-2/ICARE_NG measurements when appropriate. The time coverage considered for each spacecraft is from $20 / 06 / 2008$ to $18 / 04 / 2013$ for JASON-2, from 


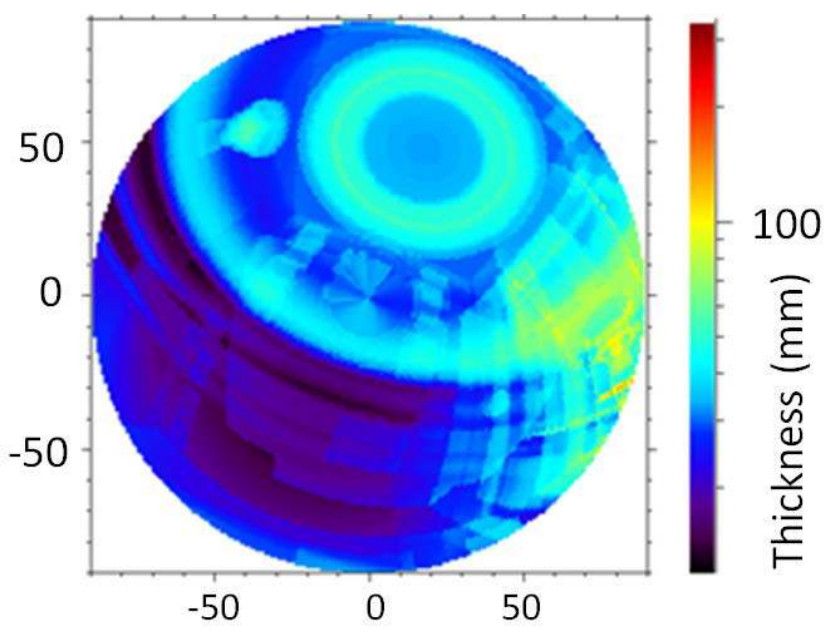

Fig. 4. Fish-eye view of JASON-2 shielding, $x$-axis and $y$-axis represent, respectively, azimuth and polar angles in the range $\left[-90^{\circ},+90^{\circ}\right]$.

$12 / 08 / 2013$ to $10 / 11 / 2013$ for SAT-X, and from 20/09/2013 up to $21 / 08 / 2015$ for SPRINT-A.

The average trapped proton environment is shown in Fig. 3(a) at Jason-2, Fig. 3(b) at SPRINT-A, and Fig. 3(c) at SAT-X orbits, from AP8 min, AP9 Mean V1.30.001, and ICARE-NG measurements. One can see that the radiation constraint is the highest for SAT-X while it is very similar for SPRINT-A and JASON-2. In all three missions, AP9 Mean V1.30.001 predicts higher fluxes than AP8 min and ICARENG measurements. Note that in Fig. 3(b), the trapped proton environment at SPRINT-A is deduced from JASON-2/ ICARE_NG measurements.

\section{Transport of Fluxes Through the Shielding}

The fluxes calculated previously for all the missions have been transported across the shielding provided by the equipment and satellite down to the CCD 47-20 (the component at the core of the star tracker). In the case of JASON-2 satellite, the 3-D geometry of both the satellite and the star tracker was available and has been used for the calculation. In the case of SAT-X, the satellite has been represented with a simplified six-sided parallelepiped geometry. And for SPRINT-A, only the 3-D geometry of the star tracker was available. In each case, the 3-D shielding seen from the central pixel of the sensor was calculated from a sector analysis performed with FASTRAD [13] using the straight line approximation technique path method [14]. This method is the most relevant for shielding analysis in proton-dominated environments, as protons only experience low scattering as they lose energy through the matter [14]. This is not recommended for electrons that have highly scattered trajectories. A fish-eye view of the JASON-2/CCD shielding including the satellite is shown in Fig. 4. One can see in Fig. 4 that an important part of the shielding from the satellite and the payload itself is greater than several centimeters.

As can be seen in Figs. 4 and 5, the distribution of shielding thicknesses ranges from few centimeters up to more than $10 \mathrm{~cm}$. At first sight, the shielding distributions of the three

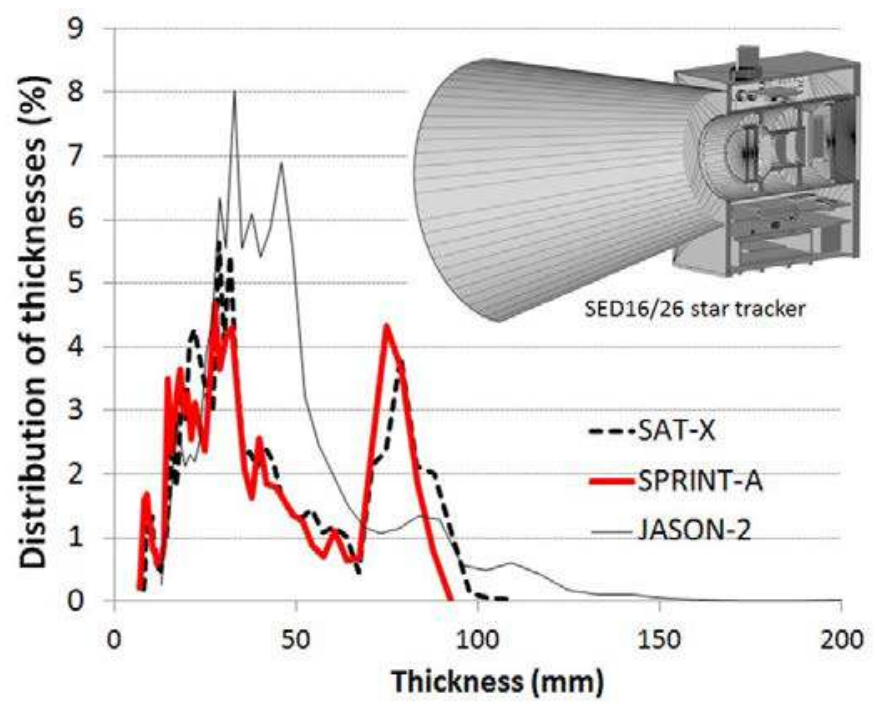

Fig. 5. Distribution of shielding thickness for the three satellites.

satellites are relatively close to each other. The shielding's distribution of the SPRINT-A satellite only takes into account the shielding of the star tracker. The distributions of the shielding of SPRINT-A and SAT-X are very close to each other, showing that the shielding of the SAT-X satellite is negligible compared to the shielding of the star tracker. The distribution of the JASON-2 satellite is slightly different, showing in that case, that the satellite platform is heavier. However, below $3 \mathrm{~cm}$, the distribution of the shielding thicknesses of JASON-2, which includes the structure of the spacecraft, is comparable to the distribution of the shielding thicknesses of the two other satellites. Despite those differences, the three distributions have nearly the same average around $\sim 5 \mathrm{~cm}$ and reach some shielding thicknesses of more than $10 \mathrm{~cm}$ (Figs. 4 and 5). In all cases, in a first approximation, the distributions of the shielding with or without taking into account the satellite structure are relatively close to each other, because the shielding of the star tracker structure is relatively important by itself (Fig. 5). In addition, the unit is located outside of the satellite; consequently, the CCD 47-20 is mainly shielded by the structure of the star tracker.

The transmitted fluxes are calculated for the central pixel of the sensor, thanks to the Monte Carlo code MCNPx V2.7.0 [15]. Depending on the satellite, the transfer's function of the shielding (Fig. 6) has been estimated in each case with or without taking into account the structure of the satellite (except for SPRINT-A where no satellite model was available).

The transmission function calculated for the SPRINT-A satellite is shown in Fig. 6. As can be seen in Fig. 6, the color codes depict a typical shape where, at high energy most of the incident particles hold a substantial part of their energy. With the decreasing energy, the part of incident particles that have lost a significant part of their energy increases. The typical shape of the transmission function is the result of a compromise between the energy of the incident protons and their capability to penetrate into matter. While the energy of the incident proton is such that its range is far longer than the thickness of the shielding, the transmitted energy 


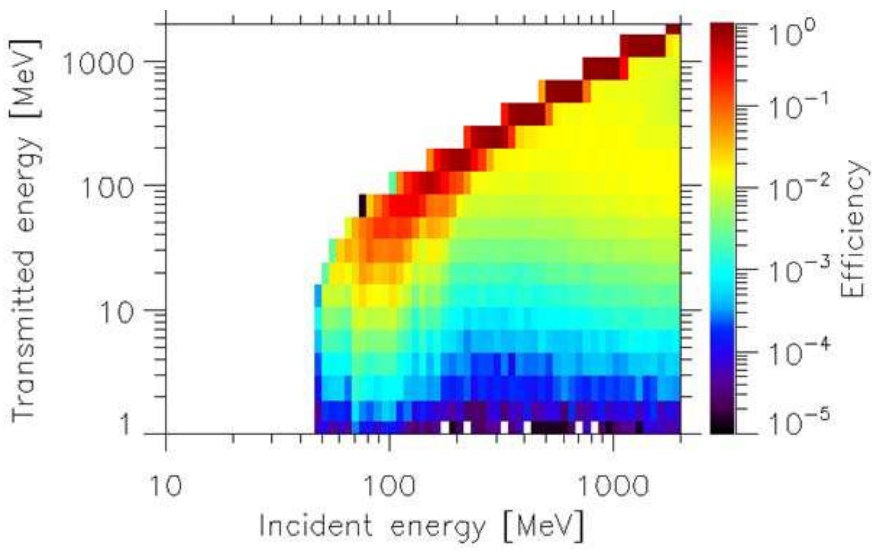

Fig. 6. Transmission functions of the incident protons through the shielding of the star tracker of the SPRINT-A satellite. Only the star tracker structure is taken into account.

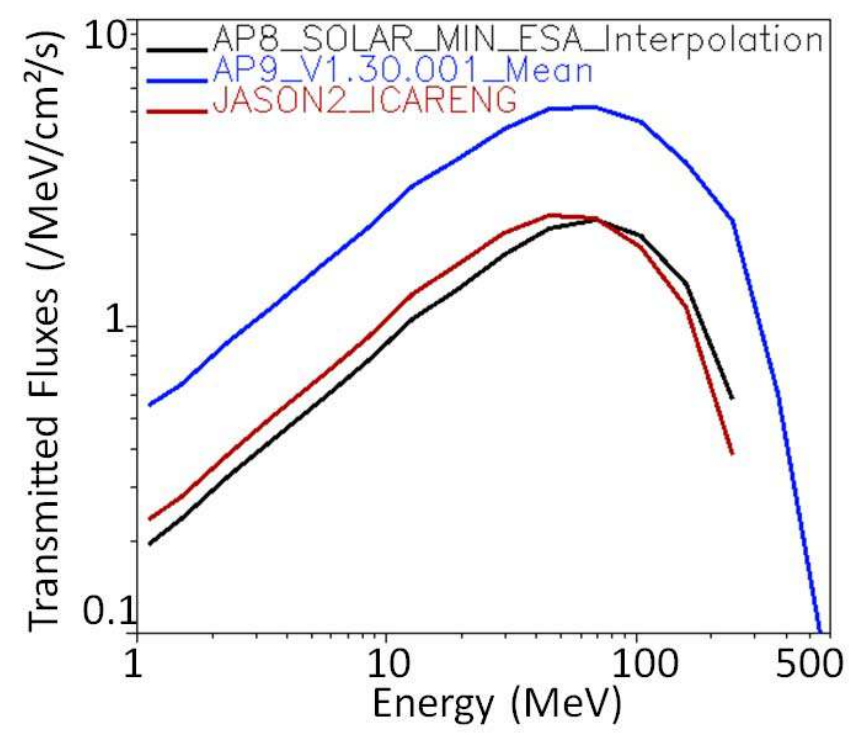

Fig. 7. Transmitted fluxes of the incident protons received by the CCD 47-20 of the SPRINT-A satellite. Only the star tracker structure is taken into account.

remains close to the incident energy. On the contrary, when the energy of the incident protons is such that, its range becomes close to the shielding thicknesses, the scatter of the transmitted energy increases significantly. For the three satellites, the transmission probability falls down to zero below $50 \mathrm{MeV}$. The transmitted fluxes have been then evaluated for the three different missions. The example of the SPRINT-A satellite is shown in Fig. 7. The energetic transmitted proton spectrum deduced from the three trapped proton environment definitions (see Section III-C) present a maximum in the range [50 MeV, $100 \mathrm{MeV}$ ] (Fig. 7).

\section{DCNU CALCUlation}

Protons produce defects in the semiconductor material by means of interactions with nuclei (Coulombic, nuclear elastic, and nuclear inelastic). These defects contribute to the increase in the dark current. Different methods exist to predict the DCNU [16]-[24]. To sum up, one can find in the literature, two main approaches. First, an analytical approach developed originally by Dale et al. [18], [19], and Marshall et al. [20] using the nonionizing energy loss (NIEL). Second, the Monte Carlo approach developed by Inguimbert et al. [16] and Ursule et al. [17]. The DCNU estimated by these two different approaches will be compared further in this paper. The principle of both methods is briefly reminded in Sections IV-A and IV-B.

\section{A. Analytical Calculation Method}

The incident protons are capable to displace atoms by means of nuclear interactions. Each recoil atoms (primary knock on atoms) initiates a damage cascade that produces a different number of atomic displacements at each time. The number of interactions is also different in each pixel, leading to a damage distribution over the array of pixels. The DCNU is then the combination of the damage distributions associated with individual interactions, weighted to the probability (Poisson) to get a given number of interactions per pixel [18]-[23]. This calculation is repeated for each kind of interactions (Coulombic, elastic and inelastic nuclear interactions). This DCNU calculation method was initially developed by Dale et al. [18], [19], and Marshall et al. [20]. It has been improved by Robbins [21], Bogaerts et al. [22], and Germanicus et al. [23]. For this paper, a simplified calculation based on the DCNU method presented earlier has been used [26]. The displacement damage dose received by the $\mathrm{CCD}$ has been converted to a monoenergetic $60-\mathrm{MeV}$ proton fluence according to NIEL factors. This particular energy was chosen as it is very close to the peak energy reaching the CCD after transport through the star tracker shielding (see Section III-D). The damage functions associated with Coulombic, elastic and inelastic interactions from $60-\mathrm{MeV}$ protons were modeled by gamma functions convolutions, with variance in damage energy obtained from [20] and weighted by Poisson statistics. Finally, the scaling factor between damage energy and dark current increase was obtained from results of displacement damage tests already performed with $60-\mathrm{MeV}$ protons.

\section{B. Monte Carlo Calculation Approach}

The second approach to predict the DCNU consists in a Monte Carlo algorithm. This more accurate approach is interesting for small geometries [17] where border crossing effect can change the shape of the DCNU. The method of prediction based on Monte Carlo algorithm is described in detail in [16] and [17]. The principle is briefly reminded here. In each pixel, the number of interactions $n$ is evaluated by a random draw in a Poisson distribution. The number of atomic displacements generated by each interaction is then evaluated, thanks to the Lindhard energy partition function for Coulombic interactions [16], [17]. For nuclear interactions which produce more energetic recoil nuclei having longer ranges than the dimensions of the pixels, the use of a Monte Carlo transport method is required. A GEANT4 [27]-[29] application has been developed to estimate the amount of damage produced in each pixel [16], [17]. The transport of the incident protons and each recoil nuclei is performed in a detailed pixel geometry. 


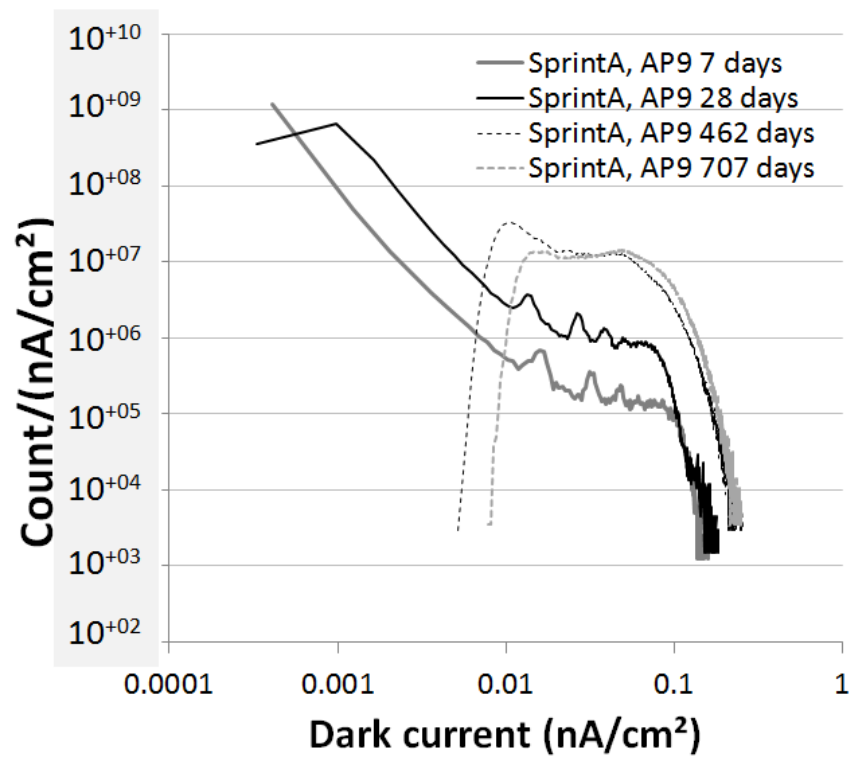

Fig. 8. Dark current distribution calculated with the Monte Carlo approach for the SPRINT-A satellite and the AP9 model. Different mission durations ranging from one week up to almost two years are presented.

The deposited damage energy is evaluated in the depleted region of the pixels of the modeled array. The damage cascades initiated outside of the depleted region, or those produced in it but capable to cross the sensitive region to get out of it, can be accurately modeled. Then the number of atomic displacements is recorded in a database for more than ten thousands of interactions [16], [17]. In a second step, this database is used to calculate the DCNU. The degradation produced by an interaction is randomly selected in this database. The damage produced in a pixel will be the sum of the damage produced by each interaction. The final damage distribution will be the combination of the damage produced by each individual interaction with the Poison's law. Finally, the scaling factor between damage energy and dark current increase was obtained from results of displacement damage tests already performed with $60-\mathrm{MeV}$ protons.

In the scope of this paper, this model originally devoted to the calculation of the DCNU induced by monoenergetic proton beams has been improved in order to take into account energetic spectra. The incident energetic distribution is splitted into 14 bins (logarithmic scale), ranging from 1 up to $\sim 250 \mathrm{MeV}$. The final DCNU is built up gradually by "adding" to the damage distribution, the damage produced by each incident energy.

\section{Examples of Calculated DCNU and $S D_{D C N U}$}

The dark current distributions of the CCD 47-20 have been estimated for different missions for increasing time durations. The classical and Monte Carlo methods of calculation of the DCNU presented in Sections IV-A and IV-B have been used. As we will see further, the two approaches give very similar results. The DCNU calculated with the Monte Carlo approach is presented in Fig. 8 for the SPRINT-A satellite using the AP9 Mean V1.30.001transmitted spectrum.

As can be seen in Fig. 8 the shape of the DCNU changes over time. It is shifted toward higher dark current values

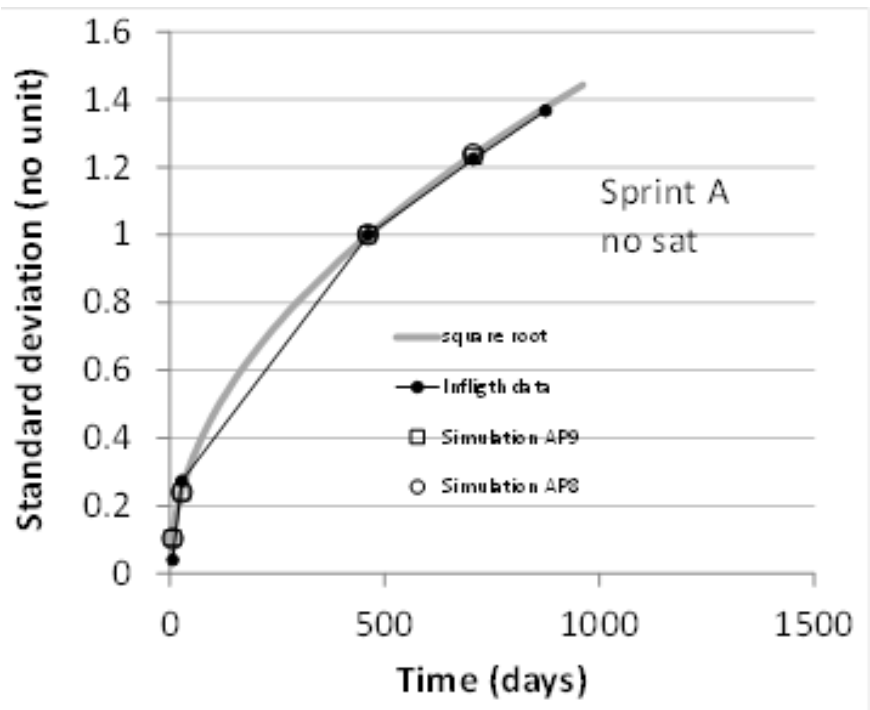

Fig. 9. SD $\mathrm{DCNU}_{\mathrm{D}}$ distribution calculated with the Monte Carlo approach for the SPRINT-A satellite. Prediction with AP8 and AP9 are compared to in-flight data. The $\mathrm{SD}_{\mathrm{DCNU}}$ has been normalized to 1 at 500 days for all the cases.

and it tends little by little toward a normal law. At low fluence level, the DCNU is a decreasing function driven by the Coulombic scattering process. The damage is dominated by the nuclear interactions only above $0.01 \mathrm{nA} / \mathrm{cm}^{2}$. When the fluence increases, according to the central limit theorem, the DCNU tends to a normal distribution with a known average and a known SD. This is observed in Fig. 8, where the peak at low damage level decreases with the time of exposure, replaced by a Gaussian distribution, representative of the damage produced by both Coulombic and nuclear interactions. For the longest integration times, the reached deposited fluences are high enough so that the Monte Carlo approach converges to the analytical approach based on the central limit theorem.

The SD ( $\left.\mathrm{SD}_{\mathrm{DCNU}}\right)$ of the dark current distributions has been calculated for different time durations, and compared to the $\mathrm{SD}$ measured in space during different missions (JASON-2, SPRINT-A, and SAT-X). The SD is demonstrated to vary accordingly to the square root of the incident total fluence. In the particular case where the proton fluxes remain almost constant along the time, the $\mathrm{SD}_{\mathrm{DCNU}}$ is then supposed to vary as a square root of the time. This is what is observed for the in-flight data. In Fig. 9, the SDDCNU of the SPRINT-A satellite is plotted as a function of time. The $\mathrm{SD}_{\mathrm{DCNU}}$ calculated numerically is comparable to in-flight measurements and follows a square root law as a function of time. In Fig. 9, for ease of comparison, the $\mathrm{SD}_{\mathrm{DCNU}}$ has been normalized to 1 at 500 days for the three environment models. Similar behaviors have been found for the three different missions. This is shown in Section V.

\section{COMParison With In-Flight Data}

\section{A. $S D_{D C N U}$}

The $\mathrm{SD}_{\mathrm{DCNU}}$ distribution has been calculated for different mission durations. This calculations have been performed 


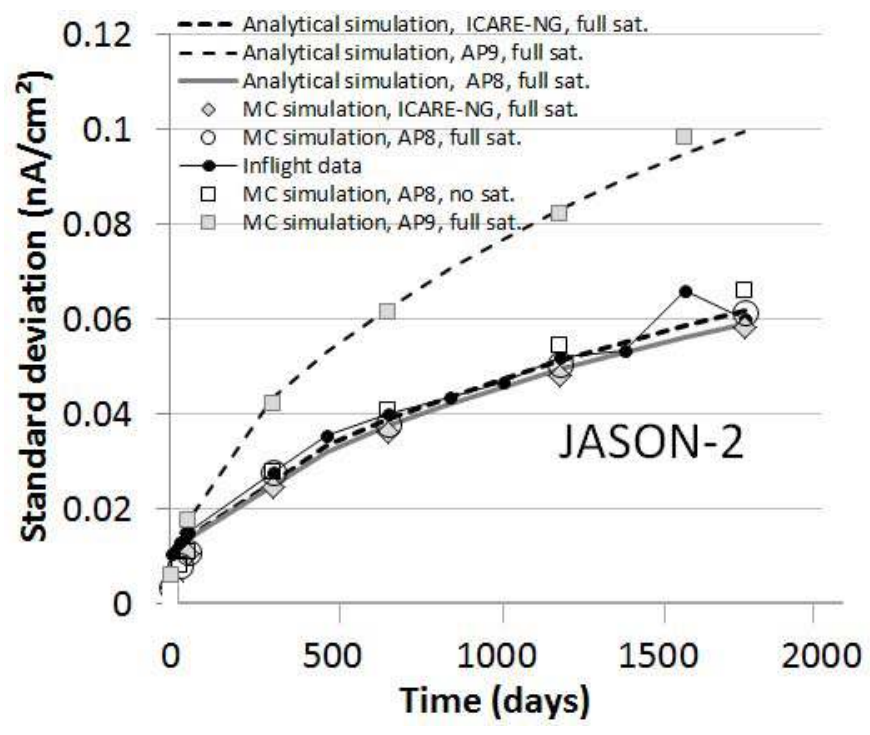

Fig. 10. JASON-2 satellite. The $\mathrm{SD}_{\mathrm{DCNU}}$ distribution calculated with both the classical and Monte Carlo approaches. The prediction made using AP8 and AP9 are compared to in-flight data and calculation performed with proton fluxes measured with the ICARE-NG radiation monitor (embedded on JASON-2).

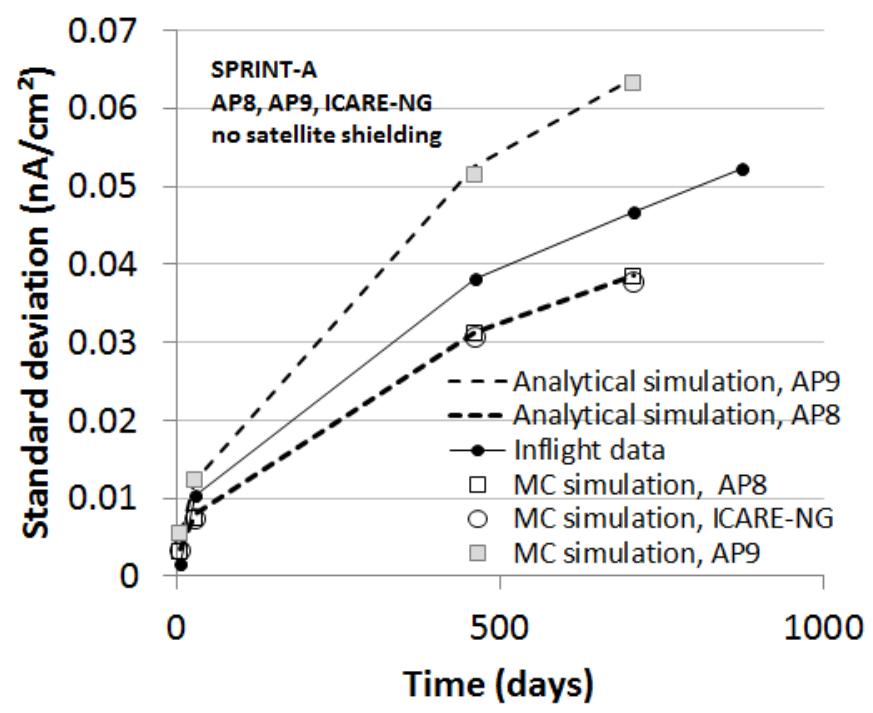

Fig. 11. SPRINT-A satellite. The SDDCNU distribution calculated with both the classical and Monte Carlo approaches. The prediction made using AP8 and AP9 are compared to in-flight data and calculation performed with proton fluxes measured with the ICARE-NG radiation monitor (embedded on JASON-2).

for the three different missions (SPRINT-A, JASON-2, and SAT-X) using AP8min and AP9 (Mean V1.30.001) environment models. The calculations have also been performed with the proton fluxes restored using the ICARE-NG radiation monitor embedded on the JASON-2 satellite. The results are compared to the in-flight data in Figs. 10-12. Both calculations made with the Monte Carlo approach and the analytical calculations are presented in Figs 10-12.

The case of JASON-2 satellite is very interesting because in that case, the proton fluxes measured onboard the spacecraft can be directly used to predict the DCNU. As can be seen

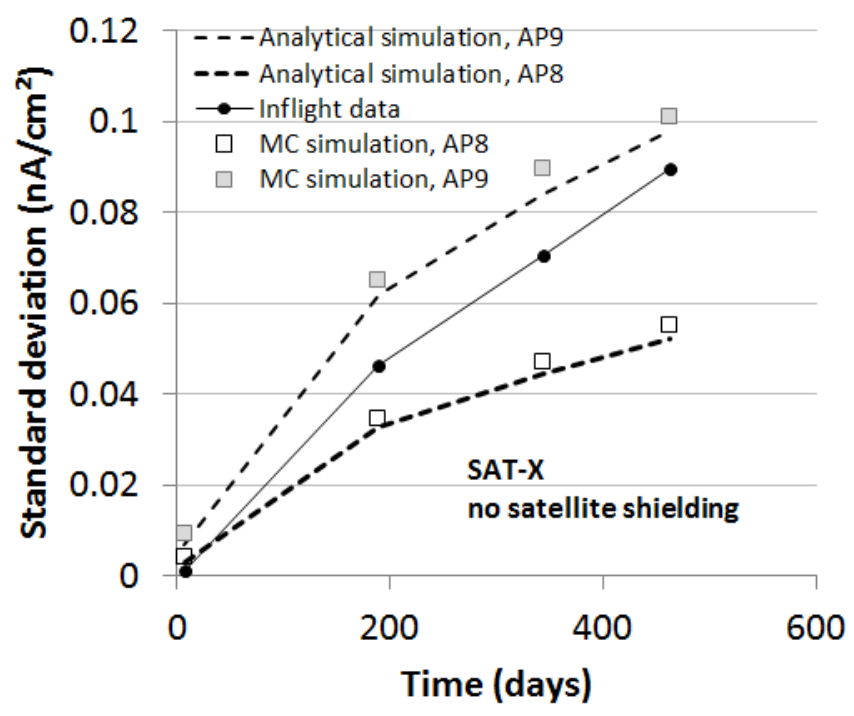

Fig. 12. SAT-X satellite. The $\mathrm{SD}_{\mathrm{DCNU}}$ distribution calculated with both the classical and Monte Carlo approaches. The prediction made using AP8 and AP9 are compared to in-flight data and calculation performed with proton fluxes measured with the ICARE-NG radiation monitor (embedded on JASON-2).

in Fig. 10, the $\mathrm{SD}_{\mathrm{DCNU}}$ simulated numerically using the protons fluxes measured onboard the JASON-2 satellite with the ICARE-NG monitor are in very good agreement with the in-flight data. And it should be noted that in that case, the $\mathrm{SD}_{\mathrm{DCNU}}$ calculated with AP8min model is also in very good agreement with the in-flight data. On the other hand the AP9 model (Mean V1.30.001) overestimates the $\mathrm{SD}_{\mathrm{DCNU}}$ distribution by nearly a factor of two. In this example (Fig. 10), neglecting the shielding of the satellite does not affect the final result. It confirms that the shielding of the image sensor is dominated by the structure of the star tracker itself.

As can be seen in Fig. 11, in the case of SPRINT-A satellite, the prediction made with the AP8min model is in good agreement with the calculation performed with the fluxes deduced from ICARE-NG radiation monitor measurements made of JASON-2 satellite. However, one can see (Fig. 11) that the $\mathrm{SD}_{\mathrm{DCNU}}$ is slightly underestimated $(\sim 20 \%)$. The prediction made with the AP9 Mean V1.30.001 model is overestimated in that case too $(\sim 40 \%)$. Those results confirm previous analysis [8], [9] performed at JASON-2 altitude where TNID measurements from an OSL sensor, and cumulated SEU monitored by an EDAC have been compared to predictions performed using AP8min and AP9 Mean V1.30.001 environment models. In that work, AP8 was found to underestimate the degradation by $20 \%$ while AP9 Mean V1.30.001 overestimates flight data by more than $100 \%$.

In the third case, (SAT-X, Fig. 12) the prediction made with the AP8min model underestimates the degradation by nearly 35\%. The AP9 Mean V1.30.001 model overestimates the $\mathrm{SD}_{\mathrm{DCNU}}$ by some $20 \%$. In this last case, the in-flight data are closer to the prediction made with the AP9 Mean V1.30.001 model than the one made with AP8min. This discrepancy could potentially be explained by a more important temporal variability of the trapped proton population at the 
outer edge of earth inner radiation belt, which is only explored by the SAT_X satellite.

\section{B. Uncertainties}

But, the observed discrepancies can be analyzed with respect to the uncertainties within the calculations. There are uncertainties at different levels. The accuracy of the calculation depends on the precision of the restitution of the orbit but also on the transport of protons through the shielding down to the CCD as well as on the DCNU calculation itself. The different parameters involved in the calculation suffer uncertainties with varying degrees of magnitude that will impact differently the precision of the final calculation. We shall concentrate here to detail how we managed to limit our sources of errors.

As already mentioned, the orbits have been estimated, thanks to the SGP4 NORAD's two lines elements propagator with a time step of $20 \mathrm{~s}$. It ensures us to fly the orbit with an accuracy of several hundred of meters minimizing the errors related to this parameter.

Concerning the transport of the protons, the main source of uncertainty depends on the accuracy of the sector analysis. The sector analysis, provided by the equipment and the satellite manufacturers, have been estimated with a solid angle precision of $1.7410^{-3}$ steradian ensuring a quite good accuracy. It consists in partitioning the solid angle viewed from the pixel in 7200 sectors of equal value, i.e., 120 steps in polar angles and 60 steps in azimuthal angles. In addition, the calculations have shown that the transport through the shielding is largely dominated by the structure of the star tracker for which an accurate description has been provided by SODERN (Fig. 5). In the case of JASON-2, the simulations performed with or without taking into account the structure of the satellite have revealed some variations of around $\sim 10 \%$ which is estimated to be a realistic error bar for the transport part of the calculation.

But the dark current calculation suffers itself some uncertainties. In this paper, the DCNU increase is considered to be mainly caused by bulk defects from displacement damage, whereas surface dark current from TID can also affect DCNU on CCDs. Thanks to the mitigation techniques used on the star tracker and presented in Section III-A, the surface component of DCNU is almost suppressed and contributes to less than $1 \%$ of the measured increase. The amount of atomic displacement produced in a pixel (NIEL [5]) is known with a relatively good accuracy, but the electrical degradations of the devices depends on the amount of produced defects subsequent to the irradiation and not to the initially estimated amount of atomic displacements. Actually, the amount of generation centers responsible of the dark current increase is strongly dependent on the annealing effects that take place after the irradiation. These phenomena are time and temperature dependent, and the annealing laws are not known with a great accuracy. The temporal dependence has been chosen to follow the annealing factor law given in [30]. After one month, the degradation is dominated by the amount of stable defects that does not evolve much more with time, limiting the uncertainty related to this parameter after this period of time. The dark current dependence on the temperature has been simulated according to an Arrhenius law, function of the activation energy. The choice of the activation energy, which is subject to a relatively large uncertainty, can affect strongly the generation current, through the exponential dependence of the dark current on this parameter. To address these two issues, and in order to reduce the uncertainties, the activation energy has been chosen in order to best fit some experimental measurements made one month after the irradiation, with $60-\mathrm{MeV}$ protons at $283 \mathrm{~K}$, the operating temperature of the device in the star tracker system. This calibration method of the DCNU calculation shows a good accuracy when applied to two different fluence levels $\left(3.3610^{+10}\right.$ and $\left.6.7210^{+10} \mathrm{p} / \mathrm{cm}^{2}\right)$.

\section{CONCLUSION}

The star trackers of the equipment manufacturer SODERN present onboard different spacecrafts have been used to perform a benchmark study of space environment models. The DCNU of the CCD 47-20 of the star tracker SED16/26 present on three different satellites (JASON-2, SPRINT-A, and SAT-X) has been evaluated for that comparative study.

The $\mathrm{SD}_{\mathrm{DCNU}}$ has been calculated as a function of time and compared to in-flight measurements. Within the measurement and calculation uncertainties, some conclusions can be drawn. The DCNU is demonstrated to evolve according to a square root of time. For the three satellites, and consistently with some previous observations, the AP9 Mean V1.30.001 model overestimates the in-flight degradation measurements. The AP8min model provides results in better agreement with flight data for satellites orbiting around $1000 \mathrm{~km}$ altitude, even though, it slightly underestimates the observations. In the case of the JASON-2 satellite, the $\mathrm{SD}_{\mathrm{DCNU}}$ calculated with the AP8min model is found to be very close to the in-flight measurements. This result is also consistent with the calculation performed with the proton fluxes measured onboard JASON-2, thanks to the ICARE-NG radiation monitor and with previous analysis. In all cases, AP8 and AP9 models represent, respectively, the lower and the upper bounds of the experimental data.

\section{REFERENCES}

[1] G. R. Hopkinson, C. J. Dale, and P. W. Marshall, "Proton effects in charge-coupled devices," IEEE Trans. Nucl. Sci., vol. 43, no. 2, pp. 614-627, Apr. 1996.

[2] C. Inguimbert and S. Messenger, "Equivalent displacement damage dose for on-orbit space applications," IEEE Trans. Nucl. Sci., vol. 59, no. 6, pp. 3117-3125, Dec. 2012.

[3] C. Inguimbert and R. Gigante, "NEMO: A code to compute NIEL of protons, neutrons, electrons, and heavy ions," IEEE Trans. Nucl. Sci., vol. 53, no. 4, pp. 1967-1972, Aug. 2006.

[4] Sodern. Sodern Neutron, Optical and Space Instrumentation. Accessed: Mar. 14, 2017. [Online]. Available: http://www.sodern.com

[5] e2v. Teledyne e2v High Performance RF Power, Semiconductor. Accessed: Mar. 14, 2017. [Online]. Available: http://www.e2v-us.com

[6] S. Bourdarie et al., "Correlation of in-flight displacement damage on the OSL sensor with space environment on-board Jason-2 spacecraft," IEEE Trans. Nucl. Sci., vol. 61, no. 4, pp. 1643-1647, Aug. 2014.

[7] S. Bourdarie et al., "Benchmarking ionizing space environment models," IEEE Trans. Nucl. Sci., vol. 64, no. 8, pp. 2023-2030, Aug. 2017.

[8] D. Falguere et al., "In-flight observations of the radiation environment and its effects on devices in the SAC-C polar orbit," IEEE Trans. Nucl. Sci., vol. 49, no. 6, pp. 2782-2787, Dec. 2002.

[9] D. Boscher et al., "In flight measurements of radiation environment on board the french satellite JASON-2," IEEE Trans. Nucl. Sci., vol. 58, no. 3, pp. 916-922, Jun. 2011. 
[10] B. Burke and S. A. Gajar, "Dynamic suppression of interface-state dark current in buried-channel CCDs," IEEE Trans. Electron Devices, vol. 38, no. 2, pp. 285-290, Feb. 1991.

[11] J. R. Janesick, "Multipinned phase charge-coupled device," U.S. Patent 4963952 A, Oct. 16, 1990.

[12] J. F. Fennell et al., "Van Allen Probes show that the inner radiation zone contains no MeV electrons: ECT/MagEIS data," Geophys. Res.Lett., vol. 42, no. 5, pp. 1283-1289, 2015.

[13] FastRad. TRAD-Expert Des Effets Des Radiations Dans le Spatial. Accessed: Mar. 14, 2017. [Online]. Available: http://www. trad.fr/FASTRAD-R.html

[14] P. Calvel et al., "Review of deposited dose calculation methods using ray tracing approximations," IEEE Trans. Nucl. Sci., vol. 55, no. 6, pp. 3106-3113, Dec. 2008.

[15] MCNP. A General Monte Carlo N-Particle (MCNP) Transport Code. Accessed: Nov. 16, 2017. [Online]. Available: https://mcnp. lanl.gov/

[16] C. Inguimbert et al., "Modeling the dark current non-uniformity of image sensors with GEANT4," IEEE Trans. Nucl. Sci., vol. 61, no. 6, pp. 3323-3330, Dec. 2014.

[17] M. C. Ursule, C. Inguimbert, and T. Nuns, "Impact of the border crossing effects on the DCNU for pixel arrays irradiated with high energy protons," IEEE Trans. Nucl. Sci., vol. 63, no. 4, pp. 2159-2167, Aug. 2016.

[18] C. J. Dale, P. W. Marshall, E. A. Burke, G. P. Summers, and G. E. Bender, "The generation lifetime damage factor and its variance in silicon," IEEE Trans. Nucl. Sci., vol. 36, no. 6, pp. 1872-1881, Dec. 1989.

[19] C. J. Dale, P. W. Marshall, and E. A. Burke, "Particle-induced spatial dark current fluctuations in focal plane arrays," IEEE Trans. Nucl. Sci., vol. 37, no. 6, pp. 1784-1791, Dec. 1990.
[20] P. W. Marshall, C. J. Dale, and E. A. Burke, "Proton-induced displacement damage distributions and extremes in silicon microvolumes charge injection device," IEEE Trans. Nucl. Sci., vol. 37, no. 6, pp. 1776-1783, Dec. 1990.

[21] M. S. Robbins, "High-energy proton-induced dark signal in silicon charge coupled devices," IEEE Trans. Nucl. Sci., vol. 47, no. 6, pp. 2473-2479, Dec. 2000.

[22] J. Bogaerts, B. Dierickx, and R. Mertens, "Enhanced dark current generation in proton-irradiated CMOS active pixel sensors," IEEE Trans. Nucl. Sci., vol. 49, no. 3, pp. 1513-1521, Jun. 2002.

[23] R. Germanicus et al., "Evaluation and prediction of the degradation of a COTS CCD induced by displacement damage," IEEE Trans. Nucl. Sci., vol. 46, no. 6, pp. 2830-2835, Dec. 2002

[24] C. Virmontois et al., "Displacement damage effects due to neutron and proton irradiations on CMOS image sensors manufactured in deep submicron technology," IEEE Trans. Nucl. Sci., vol. 57, no. 6 , pp. 3101-3108, Dec. 2010.

[25] M. Beaumel, D. Hervé, and L. Paganelli-Pradere, "Charge-coupled device proton-induced degradation observed on low earth orbit star tracker," in Proc. RADECS, 2008, pp. 370-375.

[26] S. Agostinelli et al., "Geant4-A simulation toolkit," Nucl. Instrum. Methods Phys. Res. A, Accel. Spectrom. Detect. Assoc. Equip., vol. 506, no. 3, pp. 250-303, Jul. 2003.

[27] J. Allison et al., "Geant4 developments and applications," IEEE Trans. Nucl. Sci., vol. 53, no. 1, pp. 270-278, Feb. 2006.

[28] GEANT4. Geant4: A Toolkit for the Simulation of the Passage of Particles Through Matter. Accessed: Mar. 14, 2017. [Online]. Available: http://geant4.web.cern.ch/geant4/

[29] J. R. Srour and D. H. Lo, "Universal damage factor for radiation-induced dark current in silicon devices," IEEE Trans. Nucl. Sci., vol. 47, no. 6, pp. 2451-2459, Dec. 2000 